\title{
COVID-19 Shutdown: die Sicht der Bürger*innen PERSÖNLICHE UND GESELLESCHAFTLICHE NACHTEILE
}

AUTOR*INNEN: JURGEN WILLEMS, MONIKA KNASSMÜLLER, LEA EGGENREICH, FALK EBINGER

ADMINISTRATIVE UNTERSTÜTZUNG: LISA SAUBERER

Willems, Jurgen, Monika Knassmüller, Lea Eggenreich, and Falk Ebinger. 2020. "COVID-19 Shutdown: Die Sicht Der Bürger*innen - Persönliche Und Gesellschaftliche Nachteile." OSF Preprints. June 16. doi:10.31219/osf.io/m7r4h https://doi.org/10.31219/osf.io/m7r4h

INSTITUTE FOR PUBLIC MANAGEMENT \& GOVERNANCE

WU | Vienna University of Economics and Business

Welthandelsplatz 1, A-1020 Vienna, Austria

https://www.wu.ac.at/pubmgt/institute I pubmgt@wu.ac.at 


\title{
COVID-19 Shutdown: die Sicht der Bürger*innen Persönliche und gesellschaftliche Nachteile
}

\author{
Jurgen Willems, Monika Knassmüller, Lea Eggenreich, Falk Ebinger
}

\section{ZUSAMMENFASSUNG}

Finanzierung: Die Studie ist Teil des Projektes Zusammenhalt von Bürger*innen in der Corona-Krise (COV20-039) des Institutes für Public Management \& Governance (PM\&G) | WU Wien, finanziert vom Wiener Wissenschafts-, Forschungs- und Technologiefonds I WWTF.

Zielsetzung und Design: Die Umfrage (482 in Österreich lebende Personen) erhebt in Form von zwei offenen Fragen die Wahrnehmung von persönlichen und gesellschaftlichen Nachteilen in der Zeit des COVID-19 bedingten Shutdowns. Die Daten werden im Hinblick auf Inhalt und Häufigkeit für die Gesamtgruppe sowie geschichtet nach Alter und Geschlecht analysiert. Zielsetzung ist, die direkten sozialen, wirtschaftlichen, ökologischen und politischen Folgen der Krise bzw. der zur Bewältigung gesetzten Maßnahmen zu identifizieren, und für die wissenschaftliche und politische Auseinandersetzung mit Krisensituationen aufzubereiten.

Rohdaten und ergänzende Unterlagen / Materialen sind öffentlich verfügbar (Willems 2020: https://osf.io/g8hn9/)

Erkenntnisse: Die Ergebnisse zeigen ein gewisses Auseinanderfallen der wahrgenommenen individuellen Nachtteile und der gesellschaftlichen Nachteile: Während als die drei wichtigsten persönlichen Nachteile das Social Distancing, der Alltag in der neuen Situation und die eingeschränkte (Bewegungs)freiheit genannt werden, wird auf gesellschaftlicher Ebene primär der wirtschaftliche Nachteil, Social Distancing und der Bereich Politik | Medien | Gesellschaft genannt.

Key Words: COVID, Austria, impact of crisis, individual and societal disadvantages

Weitere Informationen: Weitere Informationen zum Projekt finden sich auf der Website des PM\&G Institutes: https://www.wu.ac.at/en/pubmgt/researchandpublications/prosocial-and-societal-behavior/covid19.

\section{Kontakt:}

Alle Autor*innen sind mit dem PM\&G Institut affiliiert:

Institut für Public Management \& Governance (Website - Twitter - FIDES)

Wirtschaftsuniversität Wien | Welthandelsplatz 1, A-1020 Wien, Österreich

Email: pubmgt@wu.ac.at | Telefon: +43/1/31336-4232 


\section{Inhalt}

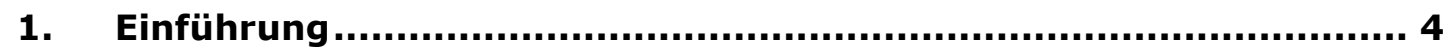

2. Forschungsdesign und Methodik.............................................. 6

3. Ergebnisse ............................................................................... 8

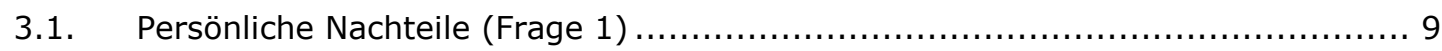

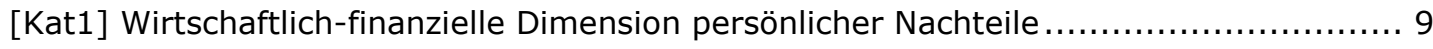

[Kat2] Alltag (beruflich \& privat) | Gesellschaftliches Klima ................................ 9

[Kat3] eingeschränkte Infrastruktur ...................................................... 10

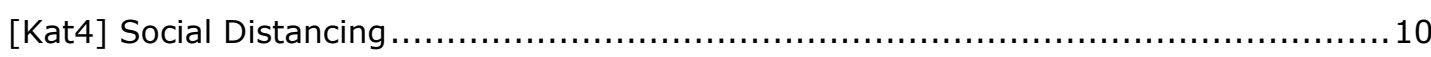

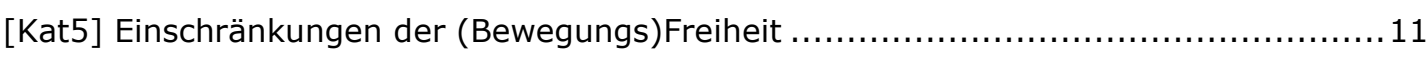

[Kat6] Gesundheitliche Dimension ....................................................... 11

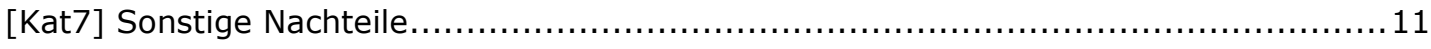

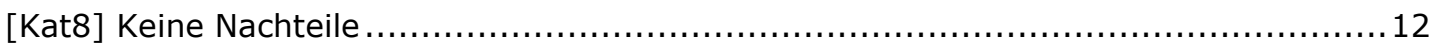

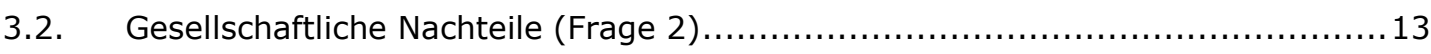

[Kat9] Wirtschaftliche Situation ........................................................ 13

[Kat10] Gesellschaftliches Klima | Politik und Medien..................................... 14

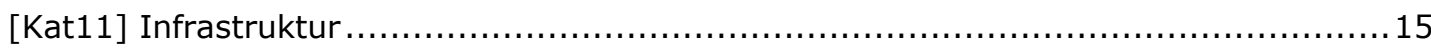

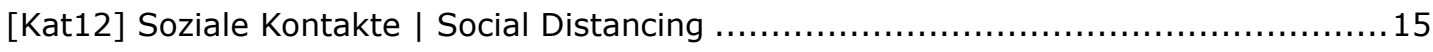

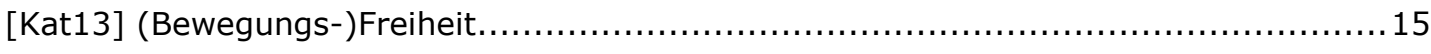

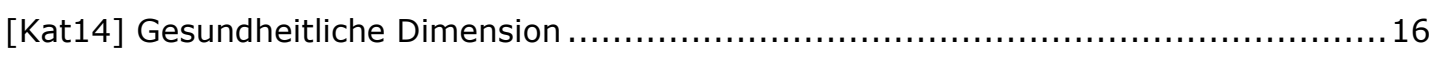

[Kat15] Sonstige gesellschaftliche Nachteile / nicht zuordenbar ...........................16

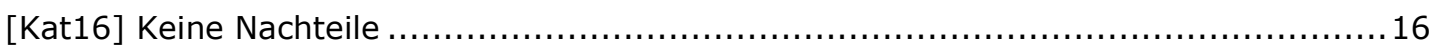

4. Limitations und Open-Access-Policy ...................................... 17

5. Literaturverzeichnis................................................................. 18

6. Anhang A: Kurzbeschreibung der Kategorien ........................... 19

7. Anhang B: Ergänzende Grafiken .............................................. 21

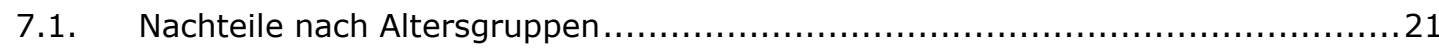

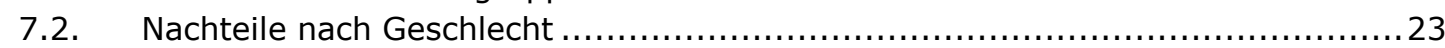




\section{Abbildungsverzeichnis}

Abbildung 1: Timeline COVID-19 Ereignisse für Österreich (bis Ende Mai 2020); (zitiert nach Europäische Kommission o. J.; ORF o. J.; wikipedia o. J.).............. 5

Abbildung 2: Verteilung Befragte nach Geschlecht und nach Altersgruppen ............... 6

Abbildung 3: Anzahl der Wörter für Frage1 (236 Befragte), Frage 2 (239 Befragte) und

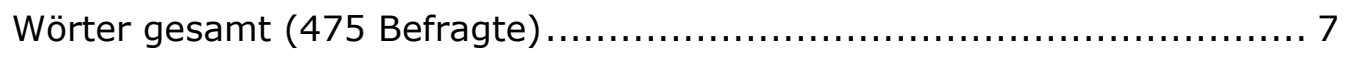

Abbildung 4: Hauptkategorien, Äußerungen pro Frage und prozentuelle Verteilung der

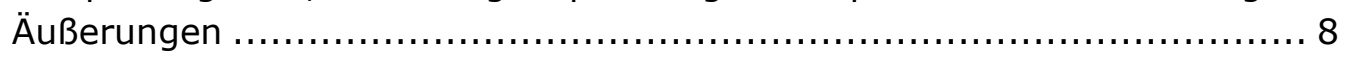

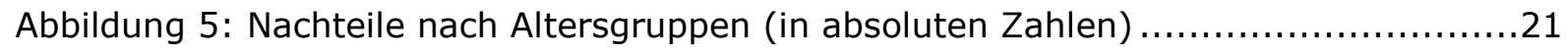

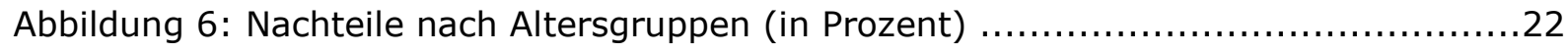

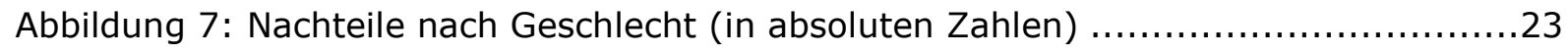

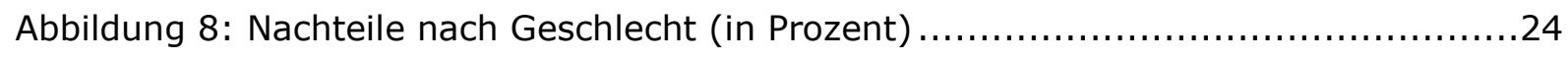

Abbildung 9: Tabellarische Timeline COVID-19 Ereignisse und Fallzahlen für Österreich (bis Ende Mai 2020); (zitiert nach Europäische Kommission o. J.; ORF o. J.; wikipedia o. J.) .............................................................. 25 


\section{Einführung}

Die österreichische Bevölkerung war in den Monaten März und April 2020 mit einer in der zweiten Republik einzigartigen Ausnahmesituation konfrontiert. Die zur Eindämmung des COVID-19-Virus ergriffenen Maßnahmen haben nicht nur massiv in den Alltag der Menschen eingegriffen, sie haben auch in psychisch-emotionaler Hinsicht kollektiv Wirkung gezeigt und Spuren hinterlassen. Die direkten sozialen, wirtschaftlichen, ökologischen und politischen Folgen der Krise bzw. der zur Bewältigung gesetzten Maßnahmen zu identifizieren und zu bearbeiten wird daher für politische Entscheidungsträger*innen ebenso wie für Wissenschafter*innen unterschiedlicher Disziplinen noch lange Priorität haben (müssen). Die Generierung von frei zugängigen Daten und Erkenntnissen (open-access-policy) hat nicht zuletzt durch die aktuelle Krisensituation enorm an Bedeutung gewonnen. Ziel des vorliegenden Berichtes ist es, zu diesen Bemühungen beizutragen.

Unsere Untersuchung erhebt die Wahrnehmung von persönlichen und gesellschaftlichen Nachteilen aus Sicht der österreichischen Bürger*innen in der Zeit des Shutdowns. Im Rahmen einer größeren Datenerhebung ${ }^{1}$ wurde zwischen 17. und 29. April 2020 einer Auswahl von 482 Befragten eine der folgenden Fragen gestellt:

(1) Was sind für Sie die größten persönlichen Nachteile aufgrund der aktuellen CoronaKrise?

(2) Was sind Ihrer Meinung nach die größten gesellschaftlichen Nachteile aufgrund der aktuellen Corona-Krise?

Die Daten bieten wertvolle Einblicke in die Belastungen, Sorgen und Befürchtungen der Bevölkerung während dieser äußerst kritischen Phase, als die Einschränkungen durch Maßnahmen zur Eindämmung des Corona-Virus vollumfänglich in Kraft waren (COVID-19Maßnahmengesetz 2020). Zugleich war die nach Ostern beginnende Phase der Lockerungen (COVID-19-Lockerungsverordnung 2020) gegen Ende der Datenerhebungsfrist zwar angekündigt, aber noch nicht konkretisiert.

\footnotetext{
${ }^{1}$ Die Studie ist Teil des Projektes Zusammenhalt von Bürger*innen in der Corona-Krise (COV20-039) des Institutes für Public Management \& Governance (WU Wien), finanziert vom Wiener Wissenschafts-, Forschungsund Technologiefonds (WWTF).
} 


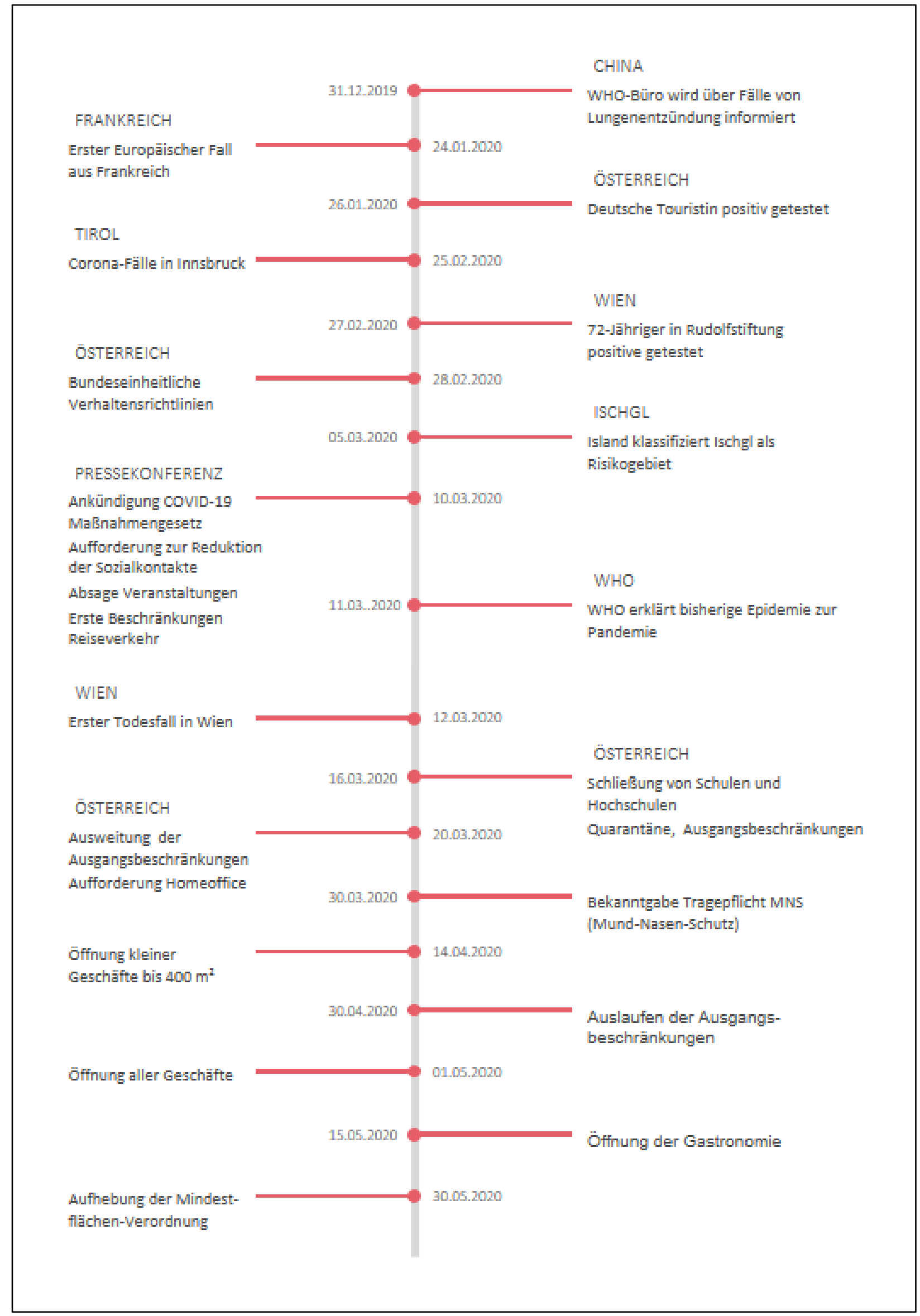

Abbildung 1: Timeline COVID-19 Ereignisse für Österreich (bis Ende Mai 2020); (zitiert nach Europäische Kommission o. J.; ORF o. J.; wikipedia o. J.) 


\section{Forschungsdesign und Methodik}

\section{Erhebungsdesign}

Die Daten wurden im Rahmen einer größeren Umfrage (siehe auch Willems u. a. 2020a; 2020b) in Form von zwei offenen Fragen erhoben: (1) Was sind für Sie die größten persönlichen Nachteile aufgrund der aktuellen Corona-Krise? und (2) Was sind Ihrer Meinung nach die größten gesellschaftlichen Nachteile aufgrund der aktuellen Corona-Krise?

Die Datenerhebung erfolgte in Form einer Online-Umfrage von 17. bis 29. April 2020, also in Woche 6 und 7 der strengsten Regierungsmaßnahmen, die als Reaktion auf die COVID19-Krise ergriffen wurden. Einer zufälligen Auswahl von 482 Umfrage-Teilnehmer*innen wurde je eine der beiden offenen Fragen gestellt. Durch die Randomisierung der Fragen in der gesamten Umfrage kann eine systematische Verzerrung durch vorhergehende Fragen ausgeschlossen werden.

\section{Datenmaterial}

Jede Frage wurde jeweils 241 Studienteilnehmer*innen gestellt, wobei nach Exkludierung leerer (4) und einer ungültigen Antwort 475 Datensätze $(F 1=239 ; F 2=236)$ in die Untersuchung einbezogen wurden. Abbildung 2 bietet eine Übersicht über die Verteilung der Befragten nach Geschlecht und Alter.

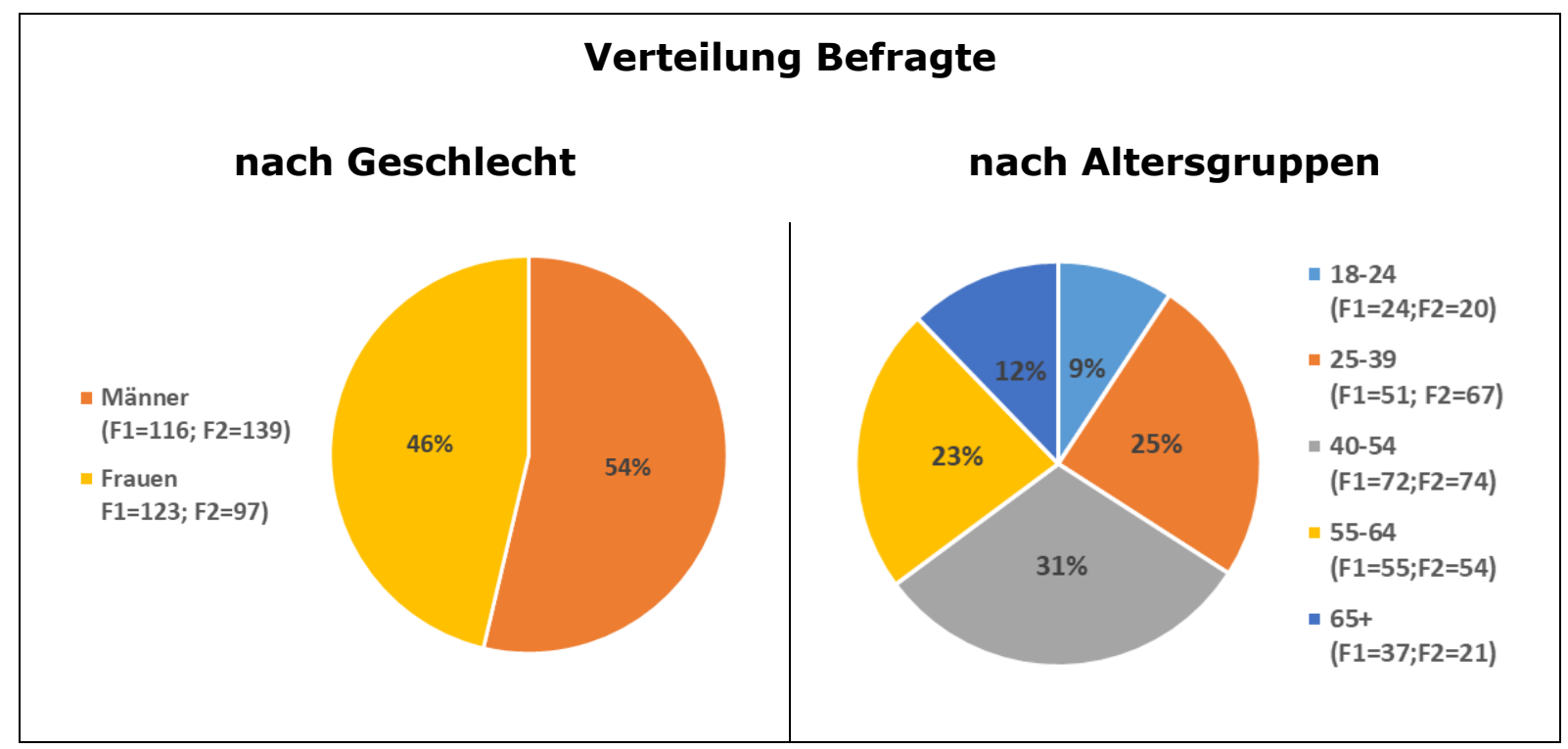

Abbildung 2: Verteilung Befragte nach Geschlecht und nach Altersgruppen

Der Großteil der Antworten (76\%) besteht aus bis zu 10 Wörtern (im Detail: $13 \%=$ ein Wort; $39 \%=$ zwei bis fünf Wörter; $23 \%=$ sechs bis 10 Wörter); 4 Befragte haben nicht geantwortet ( $1 \%$ mit 0 Wörtern). Der arithmetische Mittelwert liegt bei 9 Wörtern; der Median bei 5 Wörtern. Wie aus Abbildung 3 ersichtlich sind im Hinblick auf die beiden Fragen keine nennenswerten Unterschiede im Umfang der Antworten erkennbar. 


\section{Wörter Gesamt (475 Befragte) \\ Min $=1$ Wort $\mid$ Max $=192$ Worte $\mid$ Durchschnitt $=9$ Worte $\mid$ Median $=5$ Worte}

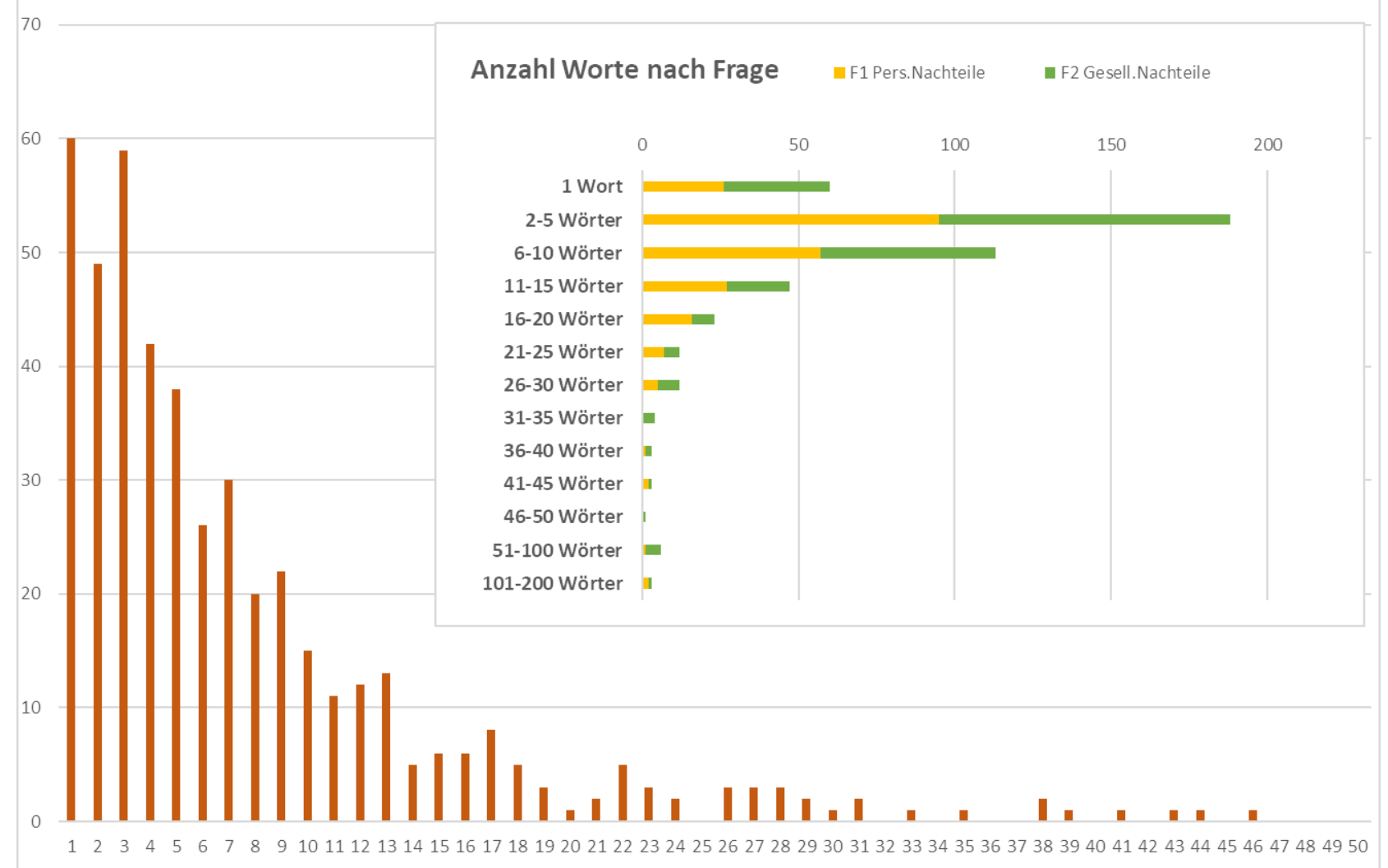

Abbildung 3: Anzahl der Wörter für Frage1 (236 Befragte), Frage 2 (239 Befragte) und Wörter gesamt (475 Befragte)

\section{Datenauswertung}

Im Rahmen einer explorativen qualitativen Inhaltsanalyse des Materials (z.B. Kuckartz 2018; Mayring 2015; Schreier 2014) wurden zunächst aus den Antworten induktiv vierzehn inhaltliche Kategorien generiert, mit denen anschließend das gesamte Material codiert wurde (eine Kurzbeschreibung der Kategorien findet sich im Anhang). Die Hauptkategorien repräsentieren thematische Dimensionen und schließen einander nicht aus. In den mehrheitlich stichwortartigen Antworten wurden häufig unterschiedliche Dimensionen angesprochen (und damit mehr als eine Hauptkategorie vergeben). 


\section{Ergebnisse}

Die beiden offenen Fragen legen unterschiedliche Perspektiven an: Während in der Frage nach persönlichen Nachteilen die eigene Betroffenheit aktiviert wird, stellt die zweite Frage auf die Beschaffenheit der Umwelt ab und richtet den Blick auf Veränderungen in dieser oder entsprechende Befürchtungen. Damit kommen in der zweiten Frage auch Vorstellungen von einer "guten" - im Sinne von wünschenswerten - Umwelt bzw. Gesellschaft zum Ausdruck, was sich auch in den konkreten Ausprägungen und Formulierungen der Antworten wiederspiegelt.

Ein erster Überblick über die induktiv generierten Hauptkategorien zur Gruppierung der Antworten zeigt eine hohe thematische Homogenität zwischen den beiden Fragen (Abbildung 4; zur Beschreibung der Kategorien siehe Anhang A). Die große Übereinstimmung in den Themengebieten lässt darauf schließen, dass die Nachteile durch die Pandemie und die damit verbundenen Maßnahmen von der Bevölkerung sehr ähnlich erlebt bzw. eingeschätzt werden.

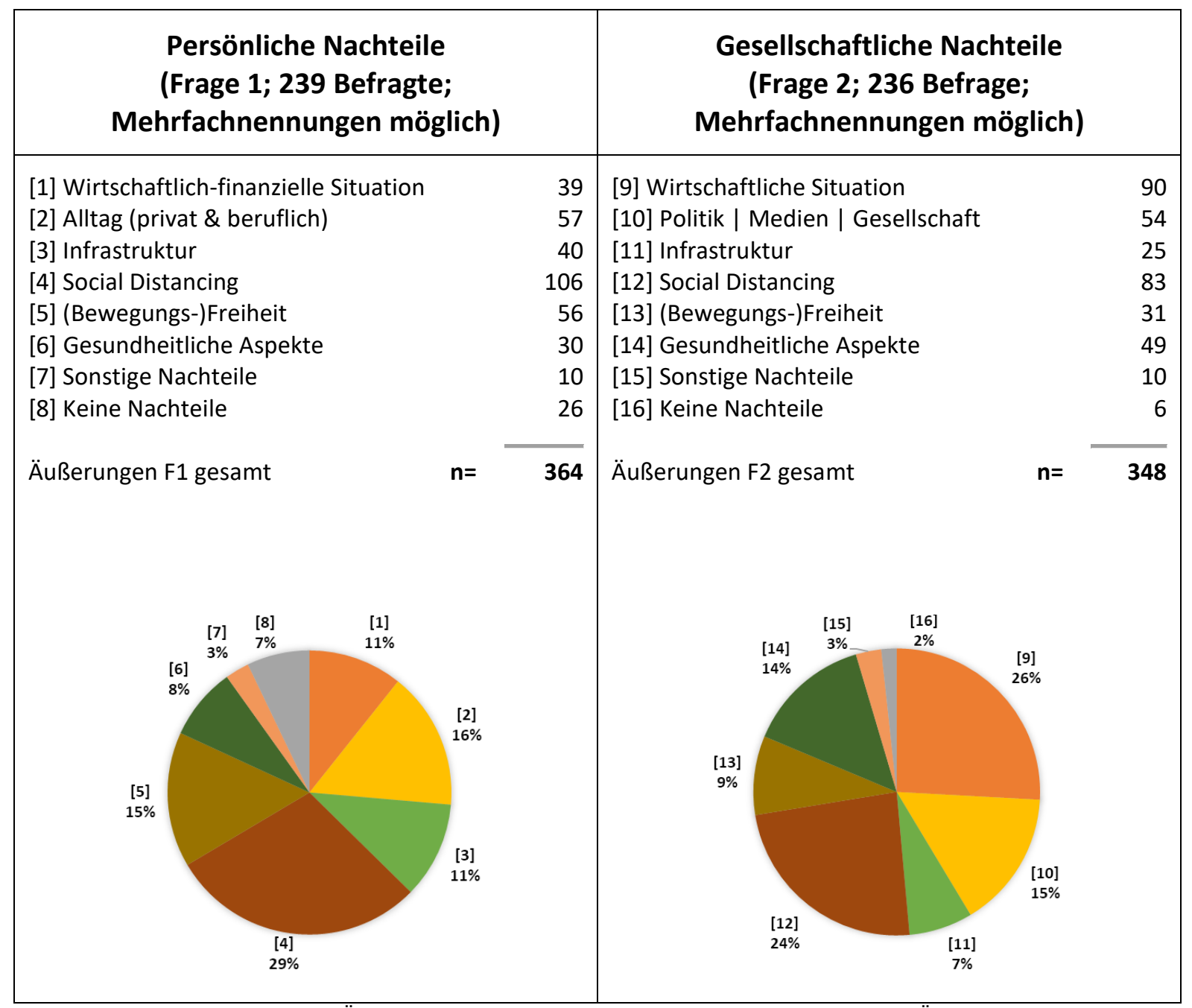

Abbildung 4: Hauptkategorien, Äußerungen pro Frage und prozentuelle Verteilung der Äußerungen 
Eindeutig perspektivenspezifisch sind die Kategorien [2] und [10]: Aussagen zum beruflichen und privaten Alltag sind ein Schwerpunkt in den Antworten zu Frage 1, während in Frage 2 die Gesellschaft - konkret: das gesellschaftliche Klima/Miteinander - eine besondere Bedeutung erfährt, ergänzt um Aussagen zu Politik und Medien. Auffällig ist auch die häufigere Nennung von wirtschaftlichen Aspekten [9] bei Frage 2. In den anderen Kategorien offenbaren sich die Unterschiede zwischen den beiden Perspektiven erst in der detaillierten Analyse der Aussagen. Im nächsten Abschnitt werden daher die Ausprägungen in den einzelnen Kategorien im Detail beschrieben. Im Anschluss daran finden sich Diskussion und Schlussfolgerungen.

\subsection{Persönliche Nachteile (Frage 1)}

F1: Was sind für Sie die größten persönlichen Nachteile aufgrund der aktuellen CoronaKrise?

In dieser Frage wird der Fokus auf die größten persönlichen Nachteile aufgrund der CoronaKrise gelenkt. Die Antworten beziehen sich daher in überwiegendem Maße auf Aspekte der persönlichen Betroffenheit bzw. Veränderungen in der unmittelbaren Lebenswelt der Befragten.

\section{[Kat1] Wirtschaftlich-finanzielle Dimension persönlicher Nachteile}

Nachteile in wirtschaftlich-finanzieller Hinsicht werden von 39 Befragten (16,3\%) thematisiert. Angesprochen werden Einkommensverluste, Verdienstentgang, Lohn- oder Gehaltseinbußen. Einkommenseffekte durch die Entwicklung auf dem Kapitalmarkt werden ein einziges Mal genannt ("meine Fonds sind stark gefallen").

Verlust oder Reduktion der unselbstständigen Erwerbsarbeit werden in 11 Antworten angeführt. Den damit einhergehenden finanziellen Konsequenzen wird durch Berücksichtigung in dieser Kategorie Rechnung getragen (die damit ebenfalls einhergehenden Auswirkungen auf den Alltag finden durch Erfassung in Kategorie 2 Berücksichtigung).

Zwei der Äußerungen thematisieren Wirtschaft auf sehr allgemeine Art ("Wirtschaft"; "das es mit der wirtschaft berg ab geht"). Da es sich um Antworten auf die Frage nach persönlichen Nachteilen handelt, wurden sie als Hinweis auf die wirtschaftlich-finanzielle persönliche Situation interpretiert und in dieser Kategorie erfasst.

\section{[Kat2] Alltag (beruflich \& privat) | Gesellschaftliches Klima}

Durch die COVID-Maßnahmen und deren Auswirkungen wurden von den Befragten auch Nachteile im beruflichen, schulischen und universitären Leben sowie in ihren privaten Lebensverhältnissen wahrgenommen. Kategorie 2 subsumiert diese Äußerungen zum privaten und beruflichen Alltagsleben sowie im zwischenmenschlichen Miteinander (gesellschaftliches Klima), sofern diese sich nicht explizit auf Social Distancing [Kat4] oder auf Einschränkung der (Bewegungs-)Freiheit [Kat5] beziehen.

Insgesamt haben 57 Befragte $(23,8 \%)$ auf die nachteilig erlebten Veränderungen im Alltagsleben Bezug genommen. Der Großteil der Aussagen (46, das sind 19,2\%) bezieht sich 
auf die Arbeits- oder Studiensituation. Davon sind 11 Personen von Arbeitslosigkeit oder Kurzarbeit betroffen (siehe auch Kategorie 1), und zwei Befragte fürchten um ihren Arbeitsplatz. Die Aussagen zur veränderten Arbeitssituation beziehen sich auf u.a. auf Arbeitszeiten, Überstunden und generelle Mehrarbeit sowie zusätzlichen Stress und Belastungen durch Homeoffice. Im Falle von Studierenden werden ein erhöhter Organisationsaufwand sowie das Fehlen von Präsenzlehre ("dass ich die Uni Vorlesungen nicht mehr besuchen kann"; "Keine Lehrveranstaltungen an der Uni"; "Einschränkungen auf der UNI") oder ausgefallene Prüfungen angeführt.

Kinderbetreuung wird in 7 Antworten, also von 2,9\% der Befragten, thematisiert, wobei zweimal die Auswirkungen durch geschlossene Kindergärten angesprochen werden. Nur eine Aussage bezieht sich explizit auf Homelearning, das dafür im Vergleich sehr umfangreich und mit Einblick in die Gefühlsebene: „Ausgeschöpfte Geduld, Kindern beim Home learning unterstützen. Verschiebung der Maturatermine, statt Sommerferien Learncamping, Urlaubsabsagen." (ID267). Sieben Antworten enthalten singuläre Äußerungen wie z.B. "Zeitmangel", "Muß selber kochen. Habe aber mehr Freizeit." oder "Vorratshaltung ist jetzt größer".

Private zwischenmenschliche Spannungen werden zweimal adressiert: "Krise in der Beziehung" und in der bereits erwähnten Aussage zum Homeschooling (",ausgeschöpfte Geduld"). Das generelle Klima im alltäglichen Leben thematisiert eine kleine Anzahl von Befragten (3), indem die Unzufriedenheit mit dem Verhalten von Mitbürger*innen zum Ausdruck gebracht wird: "Das die Leute übertreiben"; "Dass es nach wie vor viele Ignoranten und Egoisten gibt [...]"; "Keine, ich verstehe oft die Hysterie nicht. Von den Medien wird oft etwas suggeriert und labile Menschen glauben es dann."

\section{[Kat3] eingeschränkte Infrastruktur}

Den eingeschränkten Zugang zu Infrastruktur und kulturellen Veranstaltungen nannten mit 40 Befragten rund 16,7 \% als persönlichen Nachteil. Die Infrastruktur im Freizeitbereich (Sport, Kultur) wird mit 16 Äußerungen am häufigsten genannt "keine Sportmöglichkeiten"; "tennis noch nicht erlaubt"; "Schwimmbäder sind leider geschlossen"; "fehlende Kultur- Sport- und Freizeitangebote"; "Sportveranstaltungen"; "kein kulturelles Angebot"; "keine kulturellen Veranstaltungen"; "keine Veranstaltungen besuchen können"), gefolgt von Aussagen zur Schließung der Gastronomie (13): "keine Lokale besuchen können"; "würde nur gerne wieder "mein" Kaffeehaus und mein Wirtshaus besuchen können"; "Gastronomie geschlossen"; "Restaurants sind geschlossen".

Geschlossene Geschäfte werden in 8 Antworten angesprochen, wobei zwei Befragte auf das Fehlen von Produkten bzw. Hamsterkäufe Bezug nehmen. Mangelnder Zugang zum Gesundheitswesen wird in 5 Antworten angeführt: "Dass wichtige Termine bei Ärzten und in Ambulanzen abgesagt wurden"; "Keine Routineuntersuchungen beim Arzt möglich"; "keine Therapien". In zwei Fällen werden geschlossene Kindergärten thematisiert. Vereinzelte Äußerungen beziehen sich auf Dienstleistungen wie Friseur (2) und Pediküre (1) sowie die Benutzung von Öffentlichen Verkehrsmitteln (1).

\section{[Kat4] Social Distancing}

Die Dimension des sozialen Miteinanders wurde von 109 Befragten thematisiert. Der Großteil der Antworten (106 = 44,4\% der Befragten) bezieht sich auf soziale Kontakte bzw. das Vermissen solcher und ist unter [4.1 Social Distancing] zusammengefasst. Die fehlenden sozialen Kontakte zu Familie (46) und Freunden (44) werden am häufigsten genannt, 
wobei 20 Befragte beide Gruppen in ihrer Antwort erwähnen. Kolleg*innen werden nur in 3 Antworten genannt. Weitere 33 Befragte formulieren das Vermissen bzw. die Einschränkung sozialer Kontakte in allgemeiner Form, während vereinzelt (unter Diverse zusammengefasst) die fehlenden sozialen Kontakte der Kinder (2) sowie "Isolierung" (1) und "Abstand halten" (1) thematisiert werden. In drei Antworten wird im Zusammenhang mit Social Distancing die eingeschränkte Möglichkeit, anderen zu helfen, bedauert: "Das ich meine kinder nicht unterstützen kann da ich arbeiten gehen muss"; "[...] dass ich meine Freunde und meine Familie selten sehe und meine Großeltern weniger als gewohnt unterstützen kann."; "Menschen in schwierigen Lebenslagen nicht zu 100\% unterstützen zu können".

\section{[Kat5] Einschränkungen der (Bewegungs)Freiheit}

56 Befragte $(23,4 \%)$ äußerten sich zu Geboten und Verboten bzw. deren Konsequenzen im Rahmen der Covid-Maßnahmen (nicht erfasst sind hier Aussagen zur sozialen Dimension dieser Einschränkungen, die bereits in Kategorie 4 dargestellt wurden). Knapp $13 \%$ der Befragten nennen Ausgangsbeschränkungen (16) und Einschränkung der Bewegungsfreiheit (15), wobei einzelne Äußerungen eine im Vergleich zu anderen Kategorien deutlich emotionalere Sprache aufweisen: "Hausarrest", "Darf nicht raus", "Man muss zu Hause bleiben [...]". Von 11 Personen wird das Thema mit Fokus auf die persönliche Freiheit aufgegriffen: "Keine freiheit"; "Beschränkung meiner Freiheit"; "die Freiheit zu kommen und zu gehen wohin man will"; "Einschränkungen der persönlichen Freiheit", und einmal auch "freiheitsberaubung". Bürgerrechte und Demokratie werden nur von einer Person explizit angesprochen. Das Thema der Reisebeschränkungen wird in 13 Antworten adressiert.

\section{[Kat6] Gesundheitliche Dimension}

Gesundheitliche Aspekte werden von 30 Befragten (12,6\%) thematisiert.

Äußerungen zur psychisch-emotionalen Ebene betreffen zum einen Einsamkeit/Depression (8): "Ich bin völlig allein und sigmatisierte Corona Oma"; "Isolierung"; "Kein Persönlicher Kontakt mehr. / Einsamkeit"; "Man fühlt sich einsam und man bekommt Depressionen". Zum anderen werden Sorgen, Ängste und Ungewissheiten (12) zum Ausdruck gebracht, u.a. Angst vor Erkrankung / Spitalsaufenthalt, Pensionskürzung, Arbeitsplatzverlust; "Gefahrensituation"; "ungewisse Dauer der Pandemie"; "Ungewissheit wie's weitergeht"; "keine unterstützenden Therapien möglich". Auf der körperlichen Ebene werden Bewegungsmangel (4) und Gewichtzunahme (1) angesprochen.

Weitere vier Aussagen beziehen sich auf Probleme mit dem Nasen-Mund-Schutz ("Das Masken tragen, da ich keine Luft bekomme, da ich von Haus aus kurzatmig durch meine COPD bin"; "Kopfschmerz durch Maskenpflicht. Zu wenig Sauerstoff.") sowie Vorerkrankungen ("Meine Lungenerkrankung / und damit das größere Risiko") sowie (akute) "negative Auswirkungen auf psychische Gesundheit [...]".

\section{[Kat7] Sonstige Nachteile}

Zehn vereinzelte Äußerungen können wurden keiner anderen Hauptkategorie zugeordnet. Zwei davon enthalten keine sinnvolle Aussage. 
In zwei dieser Beiträge werden die Medien angesprochen: "Sehr viel Medienberichte werden gefaked oder nicht korrekt dargestellt, zu wenig Tests daher die Statistik unrealistisch"; "Keine, ich verstehe oft die Hysterie nicht. Von den Medien wird oft etwas suggeriert und labile Menschen glauben es dann."

Eine Person kritisiert die Regierung ("Die Art und Weise wie die Regierung diesen Kriese bewältigt"), drei weitere Befragte üben allgemeine Systemkritik und zeichnen ein sehr negatives Bild von der Situation, der Regierung, dem Bundespräsidenten, den Maßnahmen und deren Konsequenzen, Politikern und Lobbyisten etc. Durch die Ausführlichkeit und die teilweise Heftigkeit der Formulierungen weichen sie damit inhaltlich, sprachlich und umfänglich deutlich von den anderen Antworten ab.

Zwei Befragte bringen eine generelle Unzufriedenheit mit Mitbürger*innen zum Ausdruck: "Das die Leute übertreiben"; "Dass es nach wie vor viele Ignoranten und Egoisten gibt".

\section{[Kat8] Keine Nachteile}

Etwas mehr als $10 \%$ der Befragten (26) geben an, keine (17) oder keine nennenswerten (9) Nachteile zu haben, wobei 5 von ihnen dann doch noch welche nennen (z.B. "eigentlich keine, würde nur gerne wieder "mein" Kaffeehaus und mein Wirtshaus besuchen können."; "Ich habe keine Nachteile. Ich vermisse nur das treffen mit meinen Freunden und meiner Familie"; "Eigentlich keine. Kinderbetreuung jedoch herausfordernd"). 


\subsection{Gesellschaftliche Nachteile (Frage 2)}

F2: Was sind Ihrer Meinung nach die größten gesellschaftlichen Nachteile aufgrund der aktuellen Corona-Krise?

Diese Frage richtet den Fokus auf die größten gesellschaftlichen Nachteile aufgrund der Corona-Krise. Die Antworten beziehen sich in geringerem Ausmaß auf die unmittelbare Alltagswelt der Befragten, sondern nehmen verstärkt nachteilig wahrgenommene Veränderungen im weiteren Umfeld in den Blick. Sie geben damit Einblick in die Relevanzstrukturen der Befragten im Hinblick auf die Gesellschaft, in der sie leben möchten.

\section{[Kat9] Wirtschaftliche Situation}

Die wirtschaftliche Dimension wird von 90 Befragten $(38,1 \%)$ thematisiert.

In 15 Antworten wird die Situation der Wirtschaft generell als Nachteil angeführt: "Die wirtschaftliche Situation", "Wirtschaft", "Wirtschaftslage", "der wirtschaftliche Schaden", "wirtschaftliche Nachteile", "Wirtschaftlicher Abschwung". Etwas dramatischer formulieren 9 Befragte mit "Wirtschaftskrise" (5), "Wirtschaft wurde niedergecrasht", "wirtschaftlicher Einbruch" (2), "wirtschaftlicher Zusammenbruch", "Wirtschaftlicher Kollaps".

Finanzen, Geld und Vermögen werden in 12 Antworten $(5,1 \%)$ thematisiert. Sie beziehen sich auf wirtschaftliche Einbußen (Umsatzeinbußen, Umsatzziele, budgetierte Einnahmen) und auf die Vernichtung von Volksvermögen, Finanzwerten und den "Wertverlust von Investitionen und Vorsorgen" sowie "faule Kredite" (8). Weiters werden einmalig Deflation/Inflation, Geldentwertung und Verschuldung angesprochen sowie daraus resultierende Konsequenzen: "Finanzielle Probleme für alle später"; "Kommende Sparmaßnahmen, die jeden treffen (höhere Abgaben, bzw. Einschnitte im Sozialsystem)"; Die Gesellschaft muss jetzt all die Schulden zurückzahlen die jetzt der Finanzminister für Menschen und Firmen macht!!!!".

Die Existenzbedrohung von kleinen und mittleren Betrieben sowie als Branche insbesondere der Gastronomie werden in 9 Antworten angeführt. Eine singuläre Aussage führt das betrügerische Verhalten von Betrieben in der Krise an.

Am häufigsten - von 34 Befragten (14,4\%) werden Jobverlust und Arbeitslosigkeit als größte gesellschaftlichen Nachteile genannt, gefolgt von Äußerungen zur wirtschaftlichfinanziellen Situation der Bevölkerung (21). Letztere kreisen um finanzielle Probleme und Geldnöte, Einkommensverluste und Existenzsorgen; exemplarisch zwei etwas längere Antworten: "Wann auch die finanzielle Situation das Leben bedroht, schläft man halt nicht so gut."; "Viele Menschen haben keine finanzielle Absicherung mehr und stehen dadurch vor großen Problemen."

Von 7 Befragten wird schließlich mit Bezug auf Armut und "wirtschaftliche Ungleichheiten" die Verteilungsfrage in der Gesellschaft thematisiert: "Arme werden noch ärmer", "Verarmung eines Teiles der Bevölkerung", "sozialer Abstieg durch Arbeitslosigkeit"; in zwei Fällen wieder überdurchschnittlich lange: "Soziale Kontakte, Verständnis für gewisse Situationen, finanzielle Unterstützung für Familien und nicht für die die es eh haben sondern für die die es nicht haben."; "Es wird bald keinen Mittelstand mehr geben, sondern nur noch arm und reich. Bis jetzt haben immer nur die Profitiert die eh keine Geldsorgen haben auch schon vor der Krise". 


\section{[Kat10] Gesellschaftliches Klima | Politik und Medien}

Auf die Gesellschaft, Politik oder Medien beziehen sich 54 Befragte (22,9\%). Der Großteil der Äußerungen (44) bezieht sich auf das gesellschaftliche Klima bzw. Veränderungen im gesellschaftlichen Miteinander, während Politik, Medien und Kommunikation/Information in 15 Antworten thematisiert werden.

a. Das gesellschaftliche Klima im Sinne eines (sich nachteilig verändernden) gesellschaftlichen Miteinanders wird von 44 Befragten (18,6\%) angesprochen, wobei eine Person ganz allgemein feststellt: "alles hat dich verändert".

Eine Gruppe von Antworten thematisiert unmittelbar mit den Maßnahmen zusammenhängende Aspekte wie mangelnde Disziplin oder Missachtung der Maßnahmen (8) sowie die Ablehnung (2) oder Einforderung (1) der Kontrolle der Einhaltung, z.B.: "Mangelnde Disziplin"; "Leute die glauben sich nicht an Regeln halten zu müssen."; "daß sich nicht alle an die Vorgaben halten, und glauben sie wären nicht daran gebunden, weil sie ja nich Risikogruppe wären"; "Zu viel Angst, zu wenig Verantwortlichkeit, keine Disziplinen, keine allgemeine Verfolgung und Kontrolle bezüglich Beschränkungen".

Etwas genereller werden in 9 Antworten Egoismus und Ignoranz ("jeder schaut auf sich selbst"), mangelnde Rücksichtnahme ("Es wird keine Rücksicht auf die Mitmenschen gezeigt") und zunehmende Distanz konstatiert. In dieselbe Kerbe schlagen Befragte, die Misstrauen /mangelndes Vertrauen (5) sowie Neid, Missgunst und Habgier (4) feststellen, bis hin zu vereinzelten Aussagen wie "Panikmache mancher Menschen", "HYSTERIE - unnötige Hysterie" und "[...] Verschwörungstheorien [...]".

Aus der Perspektive von Zusammenhalt und Unterstützung thematisieren 12 Befragte die gesellschaftlichen Nachteile, formuliert als Wunsch oder Forderung (z.B. "[...] Wir könnten uns alle viel besser gegenseitig unterstützen"; "Jeder muss das tun, was für einen gemeinsamen Halt nötig ist. [...] Man muss die Mitmenschen beschützen und ethisch handeln"); oder als Missstand ("Vergessen auf älteren Menschen die alleine zu Hause sind"; "Das sich einige benachteiligt fühlen."; "Viele Streitigkeite und viel Diskussionen". In je einem Fall wird die Vernachlässigung der Flüchtlinge durch die EU angesprochen sowie die Ablehnung von Flüchtlingen(in drastischen Worten: "[...] Ich dachte, dass die Covid19-Krise Österreich von etlichen "Neuen" befreien wird bzw. werden die "Flüchtlinge" von selber nicht mehr kommen wollen [...]").

b. Politik (9) sowie Information-Kommunikation / Medien (7) werden von 15 Befragten $(6,4 \%)$ in Bezug auf gesellschaftliche Nachteile angeführt. Die Aussagen sind heterogen und breit gestreut, sodass keine inhaltlichen Schwerpunkte identifiziert werden können.

ÖVP bzw. Bundeskanzler werden in zwei Antworten direkt thematisiert: "die türkisen werden die nächsten wahlen gewinnen, weil sie gut krisenPR gemacht haben"; "Alles ein einziger Krampf. Danke, lieber Herr Verfassungsaushebler $K++z^{\prime \prime}$. Weiters werden Überwachung, Demokratieverlust und Diktatur durch die jetzige Regierung (1) bzw. Regierungen (1) genannt, sowie "Abhängigkeit von Agieren weniger (Politik) und Auswirkung auf Mehrere (Gesellschaft als Leidtragende)" verbunden mit der Hoffnung, "die Politik lernt aus dieser Krise und einzelne auch, damit wir beim nächsten Mal besser vorbereitet sein können (auch wenn ich hoffe, dass sich so etwas so schnell nicht mehr wiederholt!)". Zwei Befragte führen "Panikmache der Politik" und "Vertrauen der Politik ist geschwächt" an. Weitere 2 Antworten bewerten die Oppositionsparteien als oppor- 
tunistisch: "Dass politische Parteien - Oppositionsparteien - die Krise nützen, um populistisch Druck zu machen"; "Der Hass, die Angst und die Unsicherheit welche von den Oppsitionsparteien bewusst geschürt werden, um die ausgezeichnete Arbeit der Regierung schlecht zu machen. Nur politisches Kleingeld machen."

Sieben Antworten beziehen sich auf Information und Kommunikation allgemein (2), mangelnde Gesundheitstipps (1), sowie Probleme bei der Bewertung von Informationen ("Es werden Informationen verbreitet die unlogisch sind. Man weiss nicht mehr an was man glauben soll"; "viele unterschiedliche Meinungen über die Krise auch seitens der Wissenschaftler, Politiker und Ärzten"). Jeweils einmal werden soziale Medien sowie Medien "Es gibt keinen Austausch mehr und muss sich auf die Medien verlassen, was für mich keine Option ist, weil alle manipuliert sind und selber manipulieren" angesprochen.

\section{[Kat11] Infrastruktur}

Auf den eingeschränkten Zugang zu Infrastruktur gehen 25 Befragte (10,6 \%) in ihren Antworten ein, wobei keine nennenswerten Schwerpunkte zu erkennen sind.

In sechs Aussagen wird fehlender oder eingeschränkter Zugang zum Gesundheitswesen thematisiert in Bezug auf das Gesundheitswesen allgemein (1), die gesundheitliche Versorgung wie Untersuchungen, Behandlungen, Operationen (3) sowie das Besuchsverbot in Krankenanstalten und Altersheimen (2). Geschlossene Kindergärten und Schulen werden in vier Antworten angeführt. Das Veranstaltungsverbot wird von 6 Befragten als gesellschaftlicher Nachteil angeführt (einmal mit Bezug auf "Keine Feste, Feiern (Hochzeiten, Taufen usw)". Weiters genannt werden Schließungen von Betrieben/Dienstleistungen (1), der Gastronomie (2), Hotellerie (1) und Ausfall des Tourismus (2), die Schließung von Parks (1) und eingeschränkte Sportmöglichkeiten (2) sowie fehlende Online-Lehrveranstaltungen (1).

\section{[Kat12] Soziale Kontakte | Social Distancing}

Das Fehlen sozialer Kontakte wird von 83 Befragten (35,2\%) als gesellschaftlicher Nachteil genannt. Die Äußerungen beziehen sich auf Kontaktbeschränkungen (42) und Distanz/Abstand (3), oder konkreter auf die Kontakte zu Freunden (13) und Familie (19). Mit stärkerer Betonung auf die emotionale Dimension wird in 12 Antworten (soziale) Isolation angeführt. Vereinzelt werden einzelne Gruppen - alleinstehende Personen (2), ältere Menschen (3) und Kinder (1) - als besonders vulnerabel im Zusammenhang mit Social Distancing genannt.

In 6 Aussagen werden Konsequenzen des Social Distancing thematisiert, z.B.: "Isolation, auf sich alleine gestellt sein"; "Ausgeschlossen"; "soziale Verarmung und demfolgend Angszstörungen"; "Verhaltensveränderungen - Vereinsamung und daraus Verbitterung, Abstandhalten wird zur Gewohnheit"; "Menschen sind auf Sozialkontakte angewiesen. Wenn man sie zu lange zu Hause einspert werden sie orientierungslos und neigen fallweise zu unkontrolliertem Alkohol-Drogenkonsum. Aggressives Verhalten ist allgemein zu erwarten."

\section{[Kat13] (Bewegungs-)Freiheit}

31 Befragte $(13,1 \%)$ äußern sich zu Geboten und Verboten bzw. deren Konsequenzen im Rahmen der Covid-Maßnahmen (analog zu Kategorie 5 sind hier Aussagen zur sozialen 
Dimension dieser Einschränkungen, die bereits in Kategorie 12 dargestellt wurden, nicht erfasst). Dazu zählen Aussagen zur Einschränkung der Bewegungsfreiheit, zur Reduktion der persönlichen Freiheitsgrade, aber etwa auch zur Maskenpflicht.

In $7 \%$ der Antworten werden Ausgangsbeschränkungen (11) und Einschränkung der Bewegungsfreiheit (5) als gesellschaftliche Nachteile angeführt. Dabei wird vereinzelt auch auf Ausprägungen ("Leben auf engstem Raum"; "zu Enger Raum in den Wohnungen, kann nicht mal in den Park") und Konsequenzen gegangen wird: "Gewalt in den Familien"; "Einsamkeit bei allein lebenden Menschen, Stress bei größeren Familien, bei denen plötzlich alle dauernd zu Hause sind, Langeweile bei Kindern, die nicht in die Schule gehen dürfen". Von 6 Befragten wird auf die persönliche Freiheit fokussiert, und zwei weitere sprechen Bürgerrechte und Demokratie an: "Angriff auf unsere menschlichen Grundrechte [...]", "Verlust demokratischer Rechte". Die eingeschränkte Reisefreiheit wird von zwei Personen angesprochen.

\section{[Kat14] Gesundheitliche Dimension}

Gesundheitliche Aspekte werden von 49 Befragten (20,8 \%) thematisiert. Äußerungen zur psychisch-emotionalen Ebene betreffen psychische Erkrankungen allgemein (4), aber insbesondere Einsamkeit/Depression (28) sowie Ungewissheit, Unsicherheit und (Zukunfts)Ängste (21). Die Antworten sind mehrheitlich sehr kurz und nicht weiter konkretisiert. Die körperliche Ebene wird nur in 3 Aussagen indirekt angesprochen ("Höhe Sterblichkeit"; "Quarantäne [...]", "Krankheit"). In je einer Antwort werden die fehlenden Medikamente adressiert ("Unkenntnis über das Virus, und seiner Gefahren! / Keine heilenden Medikamente bzw. Impfstoff!"), sowie besonders negative Konsequenzen prognostiziert: "Menschen sind auf Sozialkontakte angewiesen. Wenn man sie zu lange zu Hause einsperrt werden sie orientierungslos und neigen fallweise zu unkontrolliertem Alkohol-Drogenkonsum. Aggressives Verhalten ist allgemein zu erwarten.".

Auch in dieser Kategorie werden vulnerable Gruppen benannt, insbesondere Alleinstehende (5) und ältere Menschen (1) im Zusammenhang mit Einsamkeit sowie Risikogruppen (1).

\section{[Kat15] Sonstige gesellschaftliche Nachteile / nicht zuordenbar}

Vereinzelte Äußerungen, die keiner der obigen Kategorien zuordenbar sind, betreffen die allgemeine Situation ("Diese Situation ist wirklich schlecht"; "Zer schlecht"; "alles hat dich verändert") sowie zwei Hinweise, dass die Frage jetzt noch nicht beantwortet werden kann: "Das wird uns die Geschichte zeigen! Glaskugel schauen kann ich nicht!"; "Ich glaube, dass sich das erst zu einem späteren Zeitpunkt sagen lassen wird". 4 Befragte gaben "weiß ich nicht" an, in einer Antwort wird die Mindestsicherung für alle gefordert, und zwei Äußerungen waren nicht verständlich.

\section{[Kat16] Keine Nachteile}

6 Befragte $(2,5 \%)$ geben an, keine oder keine nennenswerten gesellschaftlichen Nachteile zu erkennen. 


\section{Limitations und Open-Access-Policy}

Der vorliegende bietet eine detaillierte Beschreibung von ausgewählten Daten einer gröBeren Studie ${ }^{2}$, eine Aufbereitung derselben auf Basis einer inhaltsanalytischen Auswertung (inklusive Häufigkeiten) sowie erste Ansätze, wie diese für weitere Analysen in Beziehung gesetzt werden können. Eine vertiefende, theoretisch basierte Auswertung der gesamten Studie erfolgt in den nächsten Wochen mit dem Ziel einer möglichst zeitnahen Publikation.

Dieser Report wird zusammen mit ergänzenden Materialien der interessierten Öffentlichkeit zur Verfügung gestellt (https://osf.io/g8hn9/). Damit erhalten Entscheidungsträger*innen in Politik und Wirtschaft ebenso wie Kolleg*innen in Wissenschaft und Forschung die Möglichkeit, die Daten für eigene (weiterführende) Analysen zu nutzen, Erkenntnisse zu generieren und damit für die Gesellschaft Nutzen zu stiften. In diesem Sinne freuen wir uns über jede Verwendung der Daten und bisherigen Ergebnisse unter Angabe der entsprechenden Zitation.

\footnotetext{
${ }^{2}$ Weitere Informationen zum Projekt Zusammenhalt von Bürger*innen in der Corona-Krise (COV20-039) finden sich auf der Website des PM\&G Institutes: https://www.wu.ac.at/en/pubmgt/researchandpublications/prosocial-and-societal-behavior/covid19.
} 


\section{Literaturverzeichnis}

COVID-19-Lockerungsverordnung. 2020. „Verordnung des Bundesministers für Soziales, Gesundheit, Pflege und Konsumentenschutz betreffend Lockerungen der Maßnahmen, die zur Bekämpfung der Verbreitung von COVID-19 ergriffen wurden (COVID-19-Lockerungsverordnung - COVID-19-LV) StF: BGBI. II Nr. 197/2020". https://www.ris.bka.gv.at/Bund/.

COVID-19-Maßnahmengesetz. 2020. „Bundesgesetz betreffend vorläufige Maßnahmen zur Verhinderung der Verbreitung von COVID-19 (COVID-19-Maßnahmengesetz), BGBI. I Nr. 12/2020". https://www.ris.bka.gv.at/Bund/.

Europäische Kommission. o. J. "Website der Europäischen Kommission, Vertretung in Österreich". Zugegriffen 14. Juni 2020. https://ec.europa.eu/austria/news/focus/corona_timeline_de.

Kuckartz, Udo. 2018. Qualitative Inhaltsanalyse: Methoden, Praxis, Computerunterstützung (preview). 4. Auflage. Grundlagentexte Methoden. Weinheim Basel: Beltz Juventa.

Mayring, Philipp. 2015. Qualitative Inhaltsanalyse: Grundlagen und Techniken. 12., überarbeitete Auflage. Weinheim Basel: Beltz Verlag.

ORF. o. J. "ORF Online Berichterstattung vom 10.03.2020". Zugegriffen 16. April 2020. https://orf.at/stories/3157262/.

Schreier, Margrit. 2014. "Varianten qualitativer Inhaltsanalyse: Ein Wegweiser im Dickicht der Begrifflichkeiten". Forum Qualitative Sozialforschung / Forum: Qualitative Social Research Vol 15 (Januar): No 1 (2014). https://doi.org/10.17169/FQS15.1.2043.

wikipedia. o. J. „COVID-19-Pandemie in Österreich". Zugegriffen 14. Juni 2020. https://de.wikipedia.org/wiki/COVID-19-

Pandemie_in_\%C3\%96sterreich\#Februar_2020.

Willems, Jurgen. 2020. "Supplementary Materials: Austrians' Satisfaction in Times of COVID-19". 29. April 2020. osf.io/g8hn9.

Willems, Jurgen, Monika Knassmüller, Falk Ebinger, Katharina Dinhof, und Moritz Schmid. 2020a. "Austria in the COVID-19 pandemic - Citizens' satisfaction with crisis measures and communication". Preprint. Open Science Framework. https://doi.org/10.31219/osf.io/y37sq.

Willems, Jurgen, Monika Knassmüller, Falk Ebinger, Katharina Dinhof, und Moritz Schmid. 2020b. "COVID-19 Pandemie in Österreich - Zufriedenheit der Bürger*innen mit Maßnahmen und Kommunikation". Preprint. Open Science Framework. https://doi.org/10.31219/osf.io/g8qhf. 


\section{Anhang A: Kurzbeschreibung der Katego- rien}

Im Folgenden sind die Kategorien kurz beschrieben, um Interessierten die Orientierung in den Daten zu erleichtern und die vorgenommene Auswertung transparent zu machen.

\section{[Kat1] Wirtschaftlich-finanzielle Situation}

Diese Kategorie umfasst Äußerungen zur finanziellen und wirtschaftlichen persönlichen Situation und damit verbundene Nachteile. Indikatoren sind z.B. finanziell, wirtschaftliche, Einkommens, Gehalt, Verdienst, Lohn. Wenn Indikatoren ohne weiteren Kontext als Antwort auf die persönlichen Nachteile angeführt werden, wird dieser Code vergeben. Die Thematisierung von Wirtschaft allgemein wird - da es sich um Antworten auf die Frage nach persönlichen Nachteilen handelt - ebenfalls als Verweis auf negative Konsequenzen für die persönliche wirtschaftlich-finanzielle Situation interpretiert.

\section{[Kat2] Alltag (beruflich \& privat)}

Diese Kategorie subsumiert Äußerungen zum privaten und beruflichen Alltagsleben, sofern diese sich nicht explizit auf die Maßnahmen zur sozialen Distanzierung (siehe [Kat4] + [Kat12]) oder zur Einschränkung der (Bewegungs)Freiheit (siehe [Kat5] +[Kat13]) beziehen. Dazu gehören sowohl Aussagen zur (geänderten) Arbeitssituation als auch zu Veränderungen im privaten Alltag wie Kinderbetreuung, mehr Kochen, Hamsterkäufe etc.

\section{[Kat3] + [Kat11] Infrastruktur}

Diese Kategorie beinhaltet Äußerungen, die den eingeschränkten Zugang zu Infrastruktur thematisieren (Gesundheitswesen, Kinderbetreuungseinrichtungen, Geschäfte, Restaurants / Lokale, Sport- und Kulturveranstaltungen etc.).

\section{[Kat4] + [Kat12] Social Distancing}

In dieser Kategorie werden Aussagen erfasst, die das Gebot zur sozialen Distanz ansprechen bzw. die Betroffenheit durch dieses Gebot im Hinblick auf soziale Kontakte. Einschränkungen durch Gebote und Verbote ohne Bezug auf die soziale Dimension werden hingegen in [Kat5] / [Kat13] erfasst.

\section{[Kat5] + [Kat13] (Bewegungs)Freiheit}

Die Kategorie erfasst Aussagen zu Pandemie-bedingten Geboten und Verboten, sofern sie nicht auf die soziale Dimension dieser Einschränkungen referenzieren (diese werden in [Kat4] erfasst). -Darunter fallen Aussagen zu Einschränkungen der Bewegungsfreiheit, zur Reduktion der persönlichen Freiheitsgrade, aber etwa auch zur Maskenpflicht. 
Die Kategorie umfasst Äußerungen mit Bezug zu psychisch-emotionalen sowie körperlichphysischen Einschränkungen oder Auswirkungen. Auf der psychisch-emotionalen Ebene zählen dazu Gefühle wie Sorgen, Angst, Einsamkeit, Bedrohung, Unsicherheit; die Gefühlsebene sollte explizit angesprochen oder doch deutlich zum Ausdruck gebracht werden. Die physische Dimension umfasst Äußerungen zu körperbezogenen Nachteilen, die (negative) gesundheitliche Auswirkungen haben (können) wie beispielsweise Gewichtszunahme. Die Auswirkungen müssen allerdings nicht explizit thematisiert werden.

\section{[7] + [15] Sonstige Nachteile}

Sammelkategorie für Aussagen, die in keine der obigen Kategorien eingeordnet werden können und somit vereinzelte Aspekte zum Ausdruck bringen (zu geringe Häufigkeit für eigene Kategorie) - also quasi die Exoten. Wenn eine Person mehrere Exoten anführt, wird nur einmal gezählt.

\section{[8] + [16] Keine Nachteile}

Diese Kategorie umfasst die zum Ausdruck gebrachte Wahrnehmung, keine Nachteile zu erleiden, unabhängig davon, ob sie alleine oder als eine von mehreren Aussagen getätigt wird.

\section{[Kat9] - Wirtschaftliche Dimension}

In dieser Kategorie werden Äußerungen zusammengefasst, die Aspekte der Wirtschaft(slage) ebenso wie (erwartete, befürchtete) wirtschaftliche Folgen der Covid-Maßnahmen thematisieren. Diese Äußerungen können sich auf unterschiedliche Ebenen oder Akteure beziehen: den Staat, Unternehmen, Branchen, die Bevölkerung oder Teile davon etc. Indikatoren sind Worte wie Wirtschaft, ökonomische Lage, finanzielle Situation, Arbeitslosigkeit. Wenn sie ohne weiteren Kontext vorkommen, werden sie als Äußerungen zu einer als nachteilig wahrgenommenen wirtschaftlichen Lage im Allgemeinen gewertet und mit [Kat9] codiert.

\section{[Kat10] Gesellschaftliches Klima | Politik | Me- dien}

Diese Kategorie umfasst zum einen Äußerungen, die das gesellschaftliche Klima beschreiben. Dazu gehören Äußerungen zu wahrgenommenem oder befürchtetem Verhalten der Bevölkerung im Hinblick auf das gesellschaftliche Miteinander (mangelnde Rücksichtnahme, Ignoranz, Vertrauensverlust, fehlender Zusammenhalt), aber auch zum Stand der Gesellschaft (Demokratieverlust, Angst vor Diktatur, zu viel/zu wenig Kontrolle). Weiteres werden Aussagen zu Regierung und Medien hier erfasst. 


\section{Anhang B: Ergänzende Grafiken}

Die nachfolgenden Grafiken bieten Interessierten weitere Einblicke in die Struktur und Aussagekraft der Daten.

\subsection{Nachteile nach Altersgruppen}

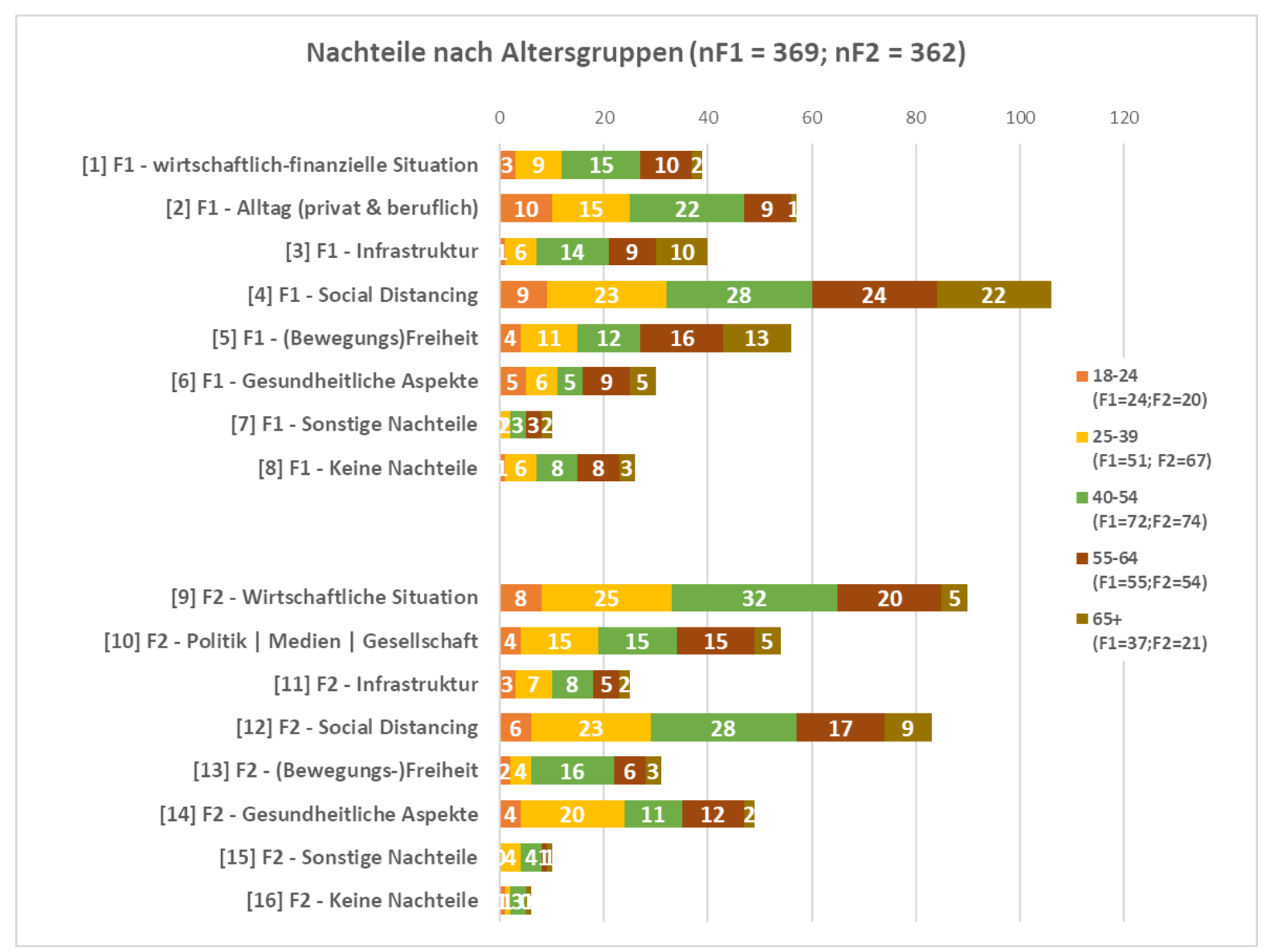

Abbildung 5: Nachteile nach Altersgruppen (in absoluten Zahlen) 


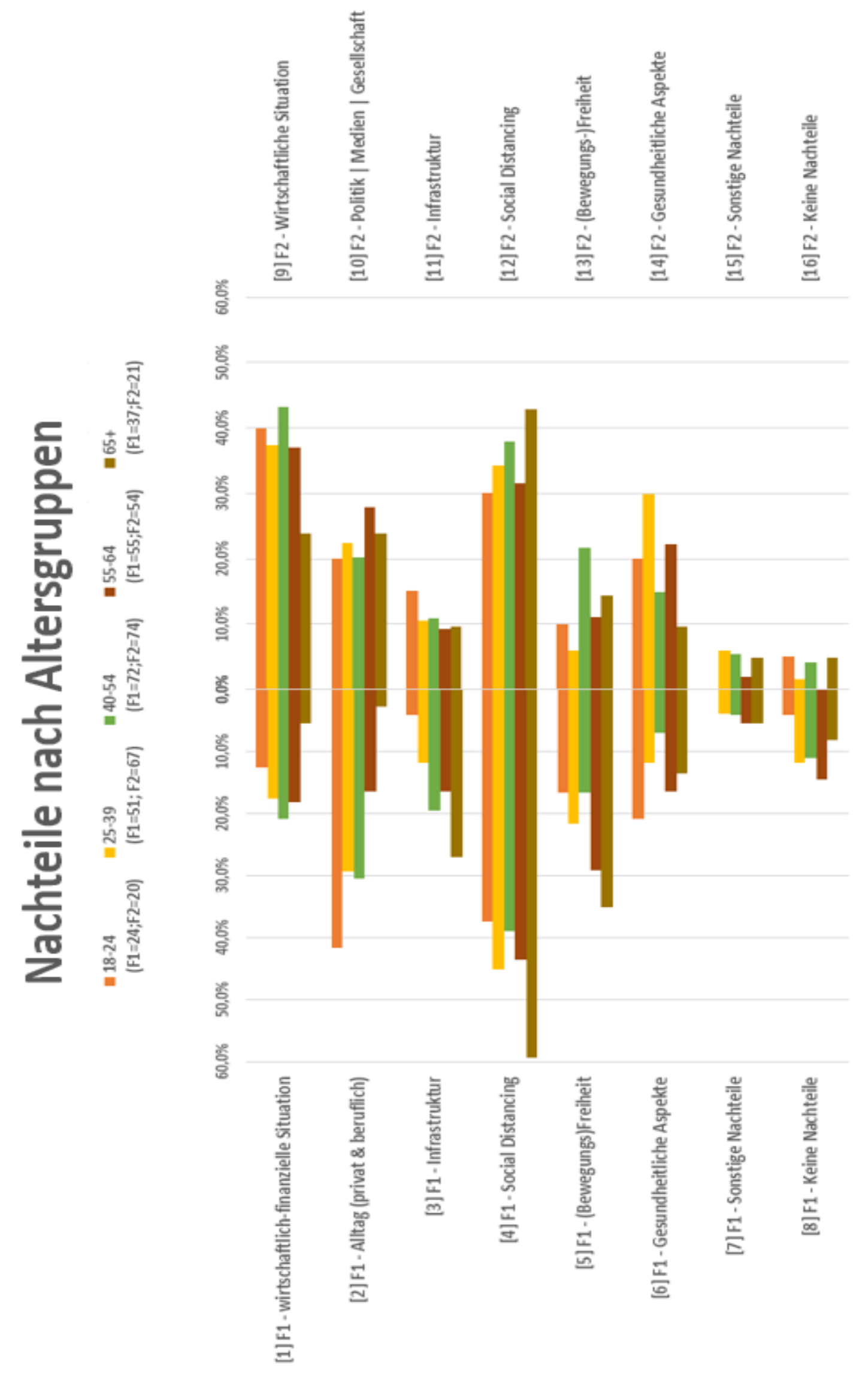

Abbildung 6: Nachteile nach Altersgruppen (in Prozent) 


\subsection{Nachteile nach Geschlecht}

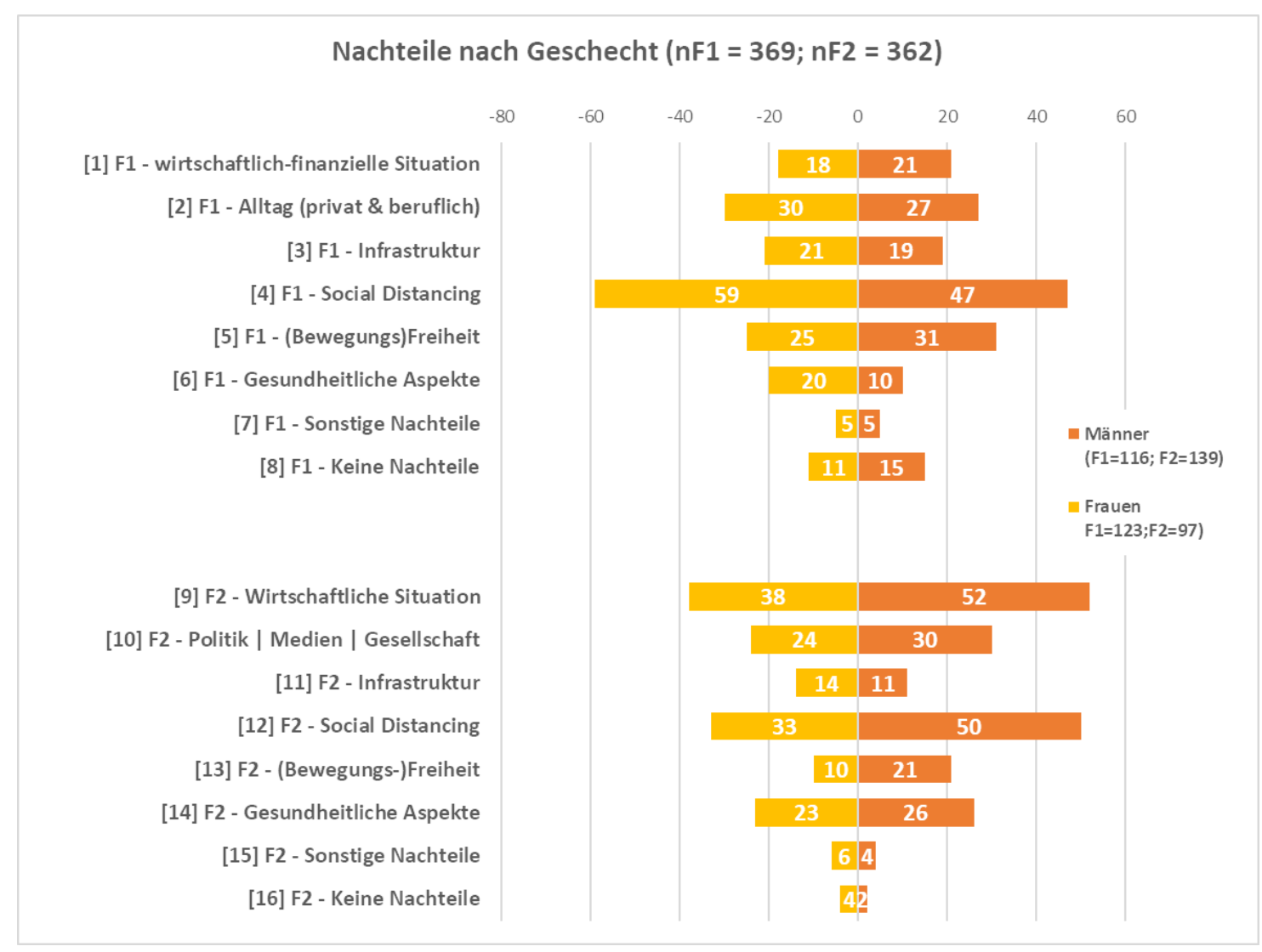

Abbildung 7: Nachteile nach Geschlecht (in absoluten Zahlen) 


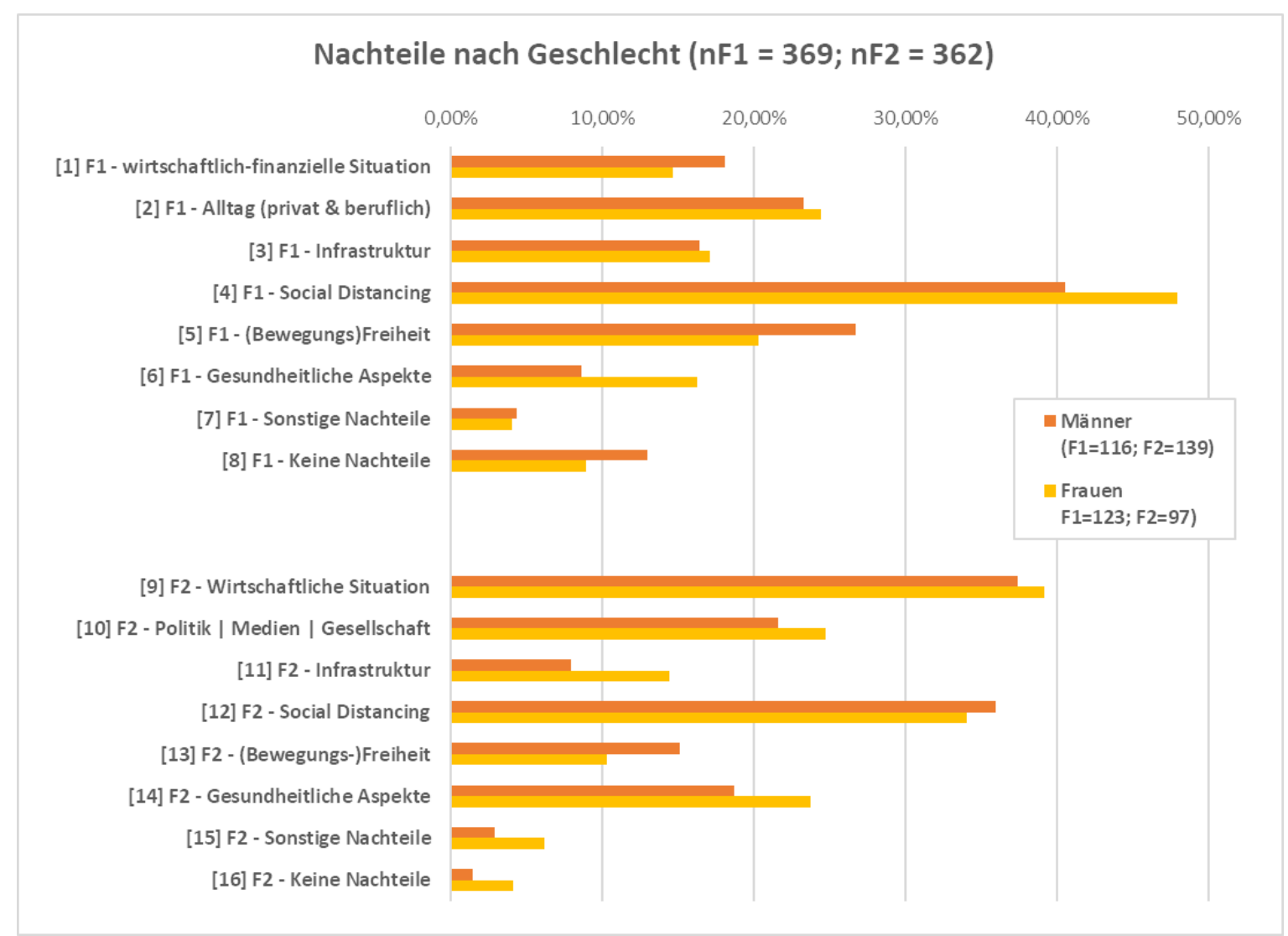

Abbildung 8: Nachteile nach Geschlecht (in Prozent) 


\subsection{Timeline (tabellarisch) und Fallzahlen Österreich}

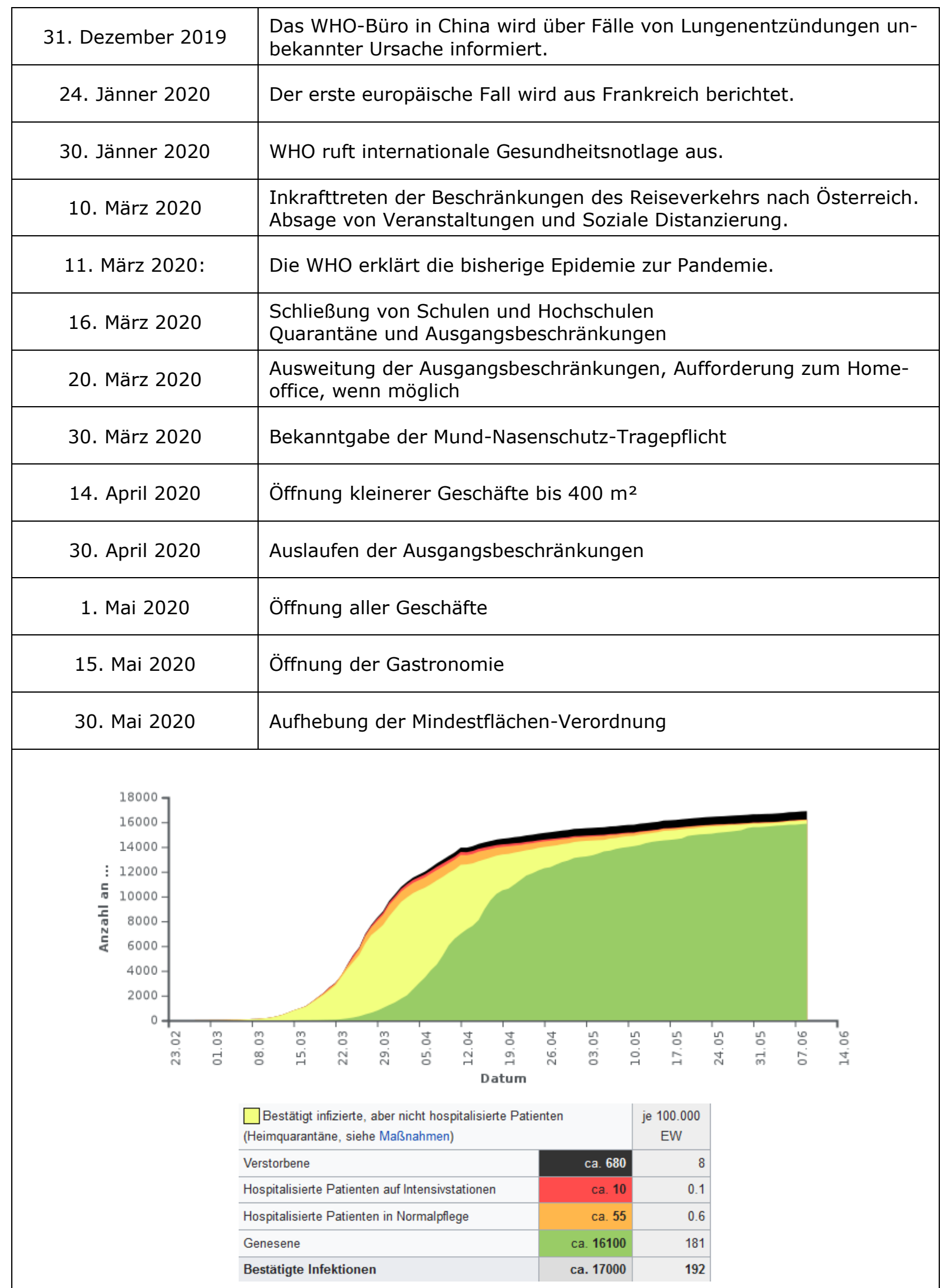

Abbildung 9: Tabellarische Timeline COVID-19 Ereignisse und Fallzahlen für Österreich (bis Ende Mai 2020); (zitiert nach Europäische Kommission o. J.; ORF o. J.; wikipedia o. J.) 